\title{
Polizeiarbeit zwischen Kontrolle und Fürsorge
}

\author{
Silvia STAUBLI \\ Universität Fribourg
}

\begin{abstract}
Einleitung
Die Polizeiarbeit hat sich in den letzten Jahrzehnten gewandelt, was z.B. die Ausbildung und die Einsatzstrategien betrifft, dies nicht zuletzt als Antwort auf gesellschaftliche Veränderungen. Darüber hinaus gibt es immer mehr verschiedene, auch private, Akteure - zum Beispiel die Bahnpolizei -, welche für die Herstellung von Sicherheit verantwortlich zeichnen. Dies birgt Konflikte im Umgang mit der Gesellschaft, aber auch zwischen den Akteuren selber. Um Herausforderungen aus Sicht solcher Akteure zu beleuchten und mögliche Problemfelder aber auch positive Aspekte zu diskutieren, fand am 22. März 2017 an der Universität Fribourg die Tagung "The Police - between Social Security and Public Safety" statt. Die folgenden Ausführungen basieren auf den gehaltenen Vorträgen. Als Erstes wird auf Veränderungen in der Polizeiarbeit verwiesen. Anschliessend wird auf zwei spezifische Strategien der Polizeiarbeit eingegangen. Als Letztes folgt die Beschreibung eines spezifischen Akteurs, nämlich der aufsuchenden Sozialarbeit am Beispiel der sip züri (Sicherheit Intervention Prävention sip züri).
\end{abstract}

\section{Veränderungen in der Polizeiarbeit}

Traditionellerweise gab es eine Distanz zwischen der Polizei und der Sozialarbeit: Repression stand Prävention gegenüber, wie Rafael Behr von der Akademie für Polizei in Hamburg für Deutschland ausführte. Diese Grenze wurde in den Folgejahren zunehmend verwischt. In den 1990er Jahren folgte auf die „Kultur der Distanzierung“ seitens der Polizei ein Paradigmenwechsel hin zu einer Kultur der „Polizeilichen Fürsorge“, welche die Bürgerpolizei und Dienstleistungen ins Zentrum stellte. Die neusten Entwicklungen weisen auf eine „Kultur der Militarisierung" hin, basierend auf einer dichotomisierten Sicht zwischen Freund und Feind und einer technologischen Aufrüstung.

Während in der traditionellen Polizei die Repression, d.h. die Strafverfolgung, dominierte und der staatliche Aspekt betont wurde, nahm nach dem Paradigmenwechsel die Bedeutung der Prävention und des Opferschutzes zu und es kam vermehrt zu einer Einzelfallorientierung. Solche Änderungen waren und sind immer eng verknüpft mit veränderten gesellschaftlichen Anforderungen. Daneben spielte die Modernisierung, gepaart mit der Feminisierung, eine wichtige Rolle. Immer modernere Geräte wie z.B. High-Tech-Einsatzfahrzeuge führten

\footnotetext{
${ }^{1}$ Silvia Staubli ist Doktorassistentin am Studienbereich Soziologie, Sozialpolitik und Sozialarbeit: sivlia.staubli@unifr.ch.

${ }^{2}$ Das Programm befindet sich im Anhang.
} 
dazu, dass die früher so wichtige körperliche Stärke an Gewicht verlor, und vermehrt Feinmotorik verlangt wird. Damit gehen eine Spezialisierung und ein erhöhter Anteil an Frauen in der Polizei einher. Die neuste Entwicklung hin zu einer Militarisierung schliesslich fusst auf der Erwartung, gegen Terrorangriffe gerüstet zu sein und rasch eingreifen zu können.

\section{Strategien der Polizeiarbeit}

Es gibt verschiedene neue oder aufbereitete Strategien, welche in den letzten Jahren in der Polizeiarbeit berücksichtigt wurden. Im Folgenden wird zum einen die bürgernahe Polizeiarbeit (community policing), zum anderen die Verfahrensgerechtigkeit (procedural justice) vorgestellt.

Unter dem Begriff des community policing werden verschiedene Aspekte zusammengefasst. Im Zentrum stehen strukturelle Anpassungen im Sinne einer Dezentralisierung, um den Bedürfnissen der Bevölkerung gerecht zu werden, die Bildung von Partnerschaften mit der Bevölkerung und anderen Institutionen, sowie eine kreative und proaktive Lösungsfindung, um die zugrundeliegenden Ursachen von Kriminalität und Unordnung zu erkennen (vgl. Bänziger 2014). Die konkrete Ausgestaltung kann sich unterscheiden, je nachdem welche Schwerpunkte im Zentrum stehen und welche Ziele man erreichen möchte.

Ein Beispiel, wie die bürgernahe Polizeiarbeit funktionieren kann, zeigte die Kantonspolizei Freiburg, vertreten durch Philippe Allain, Chef der Gendarmerie und Beat Grossrieder, Leiter des Projekts "Migranten“ und stellvertretender Leiter der bürgernahen Polizeiarbeit. Basierend auf den Säulen Präsenz, Kontakt und Partnerschaft werden unter anderem folgende Ziele verfolgt: Verminderung des Unsicherheitsgefühls bei der Bevölkerung, Absenken der Kriminalitätsrate, Erhöhung der Lebensqualität in den Quartieren sowie Herstellung und Beibehaltung des Vertrauens zwischen der Polizei und der Bevölkerung.

Den Hintergrund bildete die Flüchtlingswelle nach dem Arabischen Frühling in den Jahren nach 2010. In Fribourg äusserte sich dies in den Jahren zwischen 2012 und 2014 in einem Anstieg an Problemen und Straftaten begangen durch Asylbewerber aus Nordafrika, weshalb eine Task-Force gegründet wurde. Die ausgearbeiteten Strategien führten zu einem Rückgang der Problematik und einer verstärkten Zusammenarbeit mit Partnern aus dem strafrechtlichen, sozialen und Betreuungsbereich. Diese Erfahrungen flossen in die Strategie der Kantonspolizei Freiburg für die Betreuung der Aslyzentren im Jahr 2016 mit ein, wobei insbesondere die hohe Zahl an unbegleiteten minderjährigen Flüchtlingen (UMA) eine Herausforderung darstellte.

Vorgesehen war die „Sicherheit in Partnerschaft" durch ein massgeschneidertes Polizeidispositiv auf der einen, und eine lokale und interkantonale Zusammenarbeit auf der anderen Seite. Hierfür gab es einen kantonalen Ansprechpartner und Koordinator des „Projekt Migranten", sowie auf Seiten der Polizei einen Polizisten oder eine Polizistin als Ansprechpartner pro Asylzentrum. Nebst der Sicherstellung eines regelmässigen Kontaktes und der Analyse der Kriminalitätssituation und den Ereignissen vor Ort, sollten regelmässige Sitzungen mit den kantonalen und interkantonalen Partnern ein Weiterleiten der Informationen und eine gute Koordination von Aktionen sicherstellen. 
Ein Ziel dieses intensiven Kontaktes vor Ort in den Asylbewerberzentren ist die „Integration via Prävention (IVP)“. Anhand verschiedener Module soll nebst der Verringerung des Unsicherheitsgefühls oder der Vorbeugung von Verkehrsunfällen vor allem über Rechte und Pflichten aufgeklärt werden. In Zusammenarbeit mit Dolmetschern und unterschiedlichen Partnerorganisationen werden verschiedene Themenbereiche angesprochen: das Büro für die Gleichstellung von Frau und Mann und für Familienfragen thematisiert die Beziehung zwischen Mann und Frau sowie Belästigungen auf der Strasse; die Amtsstelle für Familienplanung und Sexualinformation thematisiert Sexualität; das Kantonale Zentrum für Abhängigkeitserkrankungen behandelt die Themen Alkohol und Drogen; die Stadt Freiburg und andere Gemeinden sprechen die Abfallentsorgung und die Prävention von Littering an; und das Jugendamt schliesslich bietet Informationen zur Staatskunde Schweiz und diskutiert Rechte und Pflichten von Bürgerinnen und Bürgern. Diese Kombination des engen Kontaktes zwischen den zuständigen Polizistinnen und Polizisten mit den Asylbewerbern und dem Vermitteln von Wissen hat sich bewährt.

Der Ansatz von procedural justice (Verfahrensgerechtigkeit) geht auf den Sozialpsychologen Tom Tyler (Tyler 1990; Tyler/Huo 2002) zurück. Im Hinblick auf die Polizeiarbeit steht hierbei die Wahrnehmung im Zentrum. Fühlt sich jemand in einer Kontrolle durch die Polizei fair und respektvoll behandelt, wirkt sich das positiv auf die Beurteilung der Polizeiarbeit und das Vertrauen in die Polizei aus. Das erhöht deren Legitimation und verbessert die Kooperation mit der Bevölkerung. Die Wirkungsmacht wurde in einer Vielzahl von Studien bestätigt. Dass aber die bewusste Anwendung von Verfahrensgerechtigkeit nicht immer zum Erfolg führt, zeigt ein Experiment aus Schottland, welches Ben Bradford von der Oxford University im Rahmen der Tagung vorstellte (vgl. MacQueen/Bradford 2015). Das Scottish Community Engagement Trial (ScotCET) war angelegt auf Strassenkontrollen während einer nationalen Kommunikationskampagne zur Strassenverkehrssicherheit über die Festtage. Involviert waren 20 Einheiten der Verkehrspolizei. Die Experimentalgruppe der Polizistinnen und Polizisten sollte gezielt Werte wie Respekt, Gleichheit, Vertrauenswürdigkeit oder Neutralität vermitteln und einen Flyer mit dem Beschrieb des Projektes aushändigen, während die Kontrollgruppe den Dienst wie üblich ausübte. Sowohl vor als auch nach der Intervention wurden Fragebögen verteilt.

Die Resultate waren ernüchternd: Die Erwartung, dass die Anwendung von Verfahrensgerechtigkeit zu einer besseren Wahrnehmung der Polizei seitens der Kontrollierten führe, wurde nicht erfüllt. Weiter noch, sie wurde ins Gegenteil verkehrt, was bedeutet, dass die Polizistinnen und Polizisten welche Verfahrensgerechtigkeit anwendeten, d.h. besonders höflich waren und die kontrollierten Verkehrsteilnehmerinnen und -teilnehmer begrüssten und verabschiedeten, schlechter bewertet wurden als Polizistinnen und Polizisten der zweiten Gruppe, welche wie bis anhin kontrollierten. War dies der Beweis, dass Verfahrensgerechtigkeit doch kein so erfolgreiches Konzept ist wie bisher angenommen? Die Nachbearbeitung des Experiments deutet in eine andere Richtung (vgl. MacQueen/Bradford 2016). Im Rahmen von Fokusgruppeninterviews mit der Experimentalgruppe kam heraus, dass folgende Faktoren zum Scheitern des Experiments führten: Erstens zeigte sich, dass das Briefing der leitenden Beamten nicht ausreichte. Es war vorgesehen, dass diese die Informationen zum Ablauf des Experiments an ihre Mitarbeitenden weiterleiten. Dies schien nicht immer der Fall gewesen 
zu sein. Aber auch bei jenen Einheiten mit guten Informationsflüssen gab es Probleme, indem gewisse Polizistinnen und Polizisten der Anweisung ihrer Vorgesetzten schlicht nicht Folge leisteten. Zweitens wurde ein gewisses Frustrationspotential erkennbar, indem gewisse Polizistinnen und Polizisten erläuterten, dass sie Aspekte der Verfahrensgerechtigkeit bereits anwenden würden und nicht verstehen, weshalb sie sich noch verbessern sollten. Es wurde drittens auch über gewisse bürokratische Aspekte gesprochen, z.B. empfand man das Aushändigen des Fragebogens im Rahmen der Routinearbeit als mühsam. Schliesslich spielte viertens der organisationale Kontext eine Rolle. Im April 2013, kurz vor der Durchführung des Experiments, kam es zu einer Zentralisierung der vormals voneinander unabhängigen Polizeieinheiten der verschiedenen Counties zu einer Police Scotland. Dieser Prozess wurde zu wenig begleitet und kommuniziert, was seitens der Polizistinnen und Polizisten zu Unsicherheit und Unzufriedenheit führte. Die Wissenschaftler folgern hieraus, dass eine engere Begleitung aller Involvierten nötig gewesen wäre, was aus Zeitgründen nicht von vorherein geplant war.

\section{Aufsuchende Sozialarbeit als Bindeglied zwischen verschiedenen Akteurinnen und Akteuren}

Mobile soziale Interventionsgruppen finden sich in der Schweiz unter anderem in den Städten Bern, Luzern, Basel-Stadt oder Zürich. An der Tagung wurde die sip züri, vertreten durch Noemi Allemann, vorgestellt. Diese mobile soziale Interventionsgruppe kombiniert aufsuchende Sozialarbeit mit Ordnungsdiensten. Sie wurde im Jahr 2000 nach der Schliessung der offenen Drogenszene am Letten ${ }^{3}$ in Zürich als Pilotprojekt gegründet. Die sozialen Dienste der Stadt Zürich realisierten damals, dass ein allein repressives Eingreifen durch die Polizei ohne die Bereitstellung von sozialen und Gesundheitsdiensten keinen Erfolg bringt und umgekehrt.

Die Politik von sip züri basiert auf den vier Säulen der Prävention, Behandlung, Schadensbegrenzung und Rechtsdurchsetzung. Die Aufrechterhaltung der öffentlichen Ordnung soll hierbei der Integration dienen. So lautet das Credo, dass der öffentliche Raum für alle zugänglich zu sein habe, mit dem Ziel der gegenseitigen Koexistenz und Toleranz. In diesem Sinne versucht die sip züri mit kommunikativen Mitteln zwischen verschiedenen Bevölkerungsgruppen zu vermitteln und handelt auf diese Weise für sozial integrierte als auch für sozial randständige Menschen.

Die Arbeit von sip züri wird oft ambivalent wahrgenommen, was sich auch in der Diskussion im Vorfeld der Abstimmung vom 12. Februar 2017 über die angepassten rechtlichen Grundlagen zeigte ${ }^{4}$. Während sip züri für die politische Linke als Kontrollorgan gilt, sind sie in den Augen der politischen Rechten überflüssig, da ihre Aufgaben auch von der Polizei übernommen werden könne. Zudem sei sip züri sowieso auf die Polizei angewiesen (sie hat keine rechtlichen Befugnisse ausserhalb der Konfliktvermittlung). Dass die Änderung der

\footnotetext{
${ }^{3}$ Der Zürcher Letten galt Anfang der neunziger Jahre als grösste offene Drogenszene Europas. Das Areal wurde Anfang 1995 geschlossen.

${ }^{4}$ Die ursprüngliche Grundlage geht auf einen Beschluss des Gemeinderates im Jahr 1990 zurück und zielte auf die Unterstützung der Drogenabhängigen. Nach der Gründung von sip züri im Jahr 2000 folgten drei Beschlüsse des städtischen Parlaments. Eine umfassende gesetzliche Verankerung, welche der veränderten gesellschaftlichen Rahmenbedingungen und der damit einhergehenden veränderten Arbeit von sip züri Rechnung trägt, fehlte jedoch.
} 
rechtlichen Grundlagen von der Bevölkerung der Stadt Zürich mit einer Mehrheit von 79.6\% angenommen wurde, zeigt jedoch auf, wie gross der Rückhalt in der Bevölkerung ist. Daneben scheint auch die Zusammenarbeit mit der Polizei sehr gut zu klappen. Gab es anfangs noch Differenzen wegen gegenseitigen Vorurteilen, so konnten diese über die Jahre hinweg abgebaut werden. Heute ist die Anzahl Anrufe, bei welchen die Polizei die sip züri aufbietet, mehr als viermal so hoch wie jene Fälle, in denen die sip züri die Polizei benötigt und diese aufbietet. Diese Zahlen unterstreichen die gute Kooperation zwischen den beiden Organisationen.

\section{Schlusswort}

Die Polizeiarbeit kommt in ihrer Tätigkeit auf der Strasse nicht nur mit verschiedenen Menschen in Berührung, sondern auch mit anderen Berufsgruppen wie Vertretern und Vertreterinnen der aufsuchenden Sozialarbeit. Auch wenn sich der Auftrag der Polizei von jenem der Sozialarbeit unterscheidet, gibt es Berührungspunkte (vgl. Simon 1999). So haben beide Professionen nicht selten mit identischen Zielgruppen zu tun. Weiter liegen die Grundlagen des beruflichen Handelns in gesellschaftlichen Defiziten, die von den Akteuren oft nicht selber verschuldet wurden. Schliesslich wird sowohl bei der Polizei als auch bei der aufsuchenden Sozialarbeit häufig nur an den Symptomen gearbeitet. Nicht zuletzt deshalb scheint eine Zusammenarbeit erfolgreich möglich, wie die Beispiele der Tagung gezeigt haben.

\section{Literaturverzeichnis}

Bänziger, Matthias (2014). Innovative Polizeiansätze. Eine kritische Begutachtung verschiedener Strategien und deren Implementierung in der Schweiz. Université de Lausanne: Thèse de doctorat, Faculté de droit et des sciences criminelles.

MacQueen, Sara und Ben Bradford (2015). Enhancing public trust and police legitimacy during road traffic encounters: results from a randomized controlled trial in Scotland. Journal of Experimental Criminology 11(3), 419-433.

MacQueen, Sara und Ben Bradford (2016). Where did it all go wrong? Implementation failure - and more - in a field experiment of procedural justice policing. Journal of Experimental Criminology, online first.

Simon, Titus (1999). Sozialarbeit und Polizei - Neue Aufgaben, Gemeinsamkeiten und notwendige Grenzen. CILIP, Bürgerrechte und Polizei, 063, 2: Jugend - Kriminalität - Polizei.

Tyler, Tom (1990). Why People Obey the Law. New Haven: Yale University Press.

Tyler, Tom R. und Yuen J. Huo (2002). Trust in the Law: Encouraging Public Cooperation with the Police and Courts. New York: Russell Sage Foundation. 


\section{Anhang}

Programm Tagung

10:15 Silvia Staubli (University of Fribourg):

„Welcome and Introduction”

10:30 Noemi Allemann (mobile intervention group sip Zurich):

„sip züri“

11:30 Discussion

12:00 Lunch break

13:30 Ben Bradford (University of Oxford, England):

"The challenges of motivating procedurally fair policing - evidence from the Scottish Community Engagement Trial”

14:30 Rafael Behr (Police Academy Hamburg, Germany):

„Von der Gerechtigkeit zur polizeilichen Fürsorge - Transformation von Polizeiarbeit in Deutschland"

15:30 Discussion

16:00 Coffee break

16:30 Philippe Allain and Beat Grossrieder (Cantonal Police of Fribourg):

„Bürgernahe Polizei”

17:30 Final discussion and wrap-up

18:00 End 